\title{
Traumatic Inferior Hip Dislocation
}

\author{
Ahmet Tugrul Zeytin ${ }^{\mathrm{a}, \mathrm{d}}$, Seyhmus Kaya ${ }^{\mathrm{a}}$, Filiz Baloglu Kaya ${ }^{\mathrm{b}}$, Hamit Ozcelik ${ }^{\mathrm{c}}$
}

\begin{abstract}
Inferior hip dislocation (luxatio erecta femoris) is the least common form of hip dislocation, and mostly occurs in children. This type of dislocation is extremely rare in adults, and there have been a total of 10 case reports in the literature. This current report presents a 55-year-old male patient who was treated with sedation analgesia and closed reduction in the emergency department with traumatic inferior hip dislocation.
\end{abstract}

Keywords: Inferior dislocation; Hip dislocation; Trauma

\section{Introduction}

High energy traumas are associated with an increased incidence of hip dislocation. Posterior hip dislocation is the most common form of hip dislocation, but inferior hip dislocations can also occur [1]. There are a very limited number of case reports in the literature and most are pediatric cases [2]. The current report presents an adult patient with inferior hip dislocation.

\section{Case Report}

A 55-year-old male patient was admitted to the emergency room after a collapsed wall fell on him. On admission, GCS was 15, and general condition was good: BP 140/70 mm Hg, PR 100 beats/min, SS 16/min, and $\mathrm{sPO}_{2} 100 \%$. On physical

\footnotetext{
Manuscript accepted for publication April 15, 2015

aDepartment of Emergency Medicine, Eskisehir State Hospital, Eskisehir, Turkey

${ }^{b}$ Department of Emergency Medicine, Eskisehir Osmangazi University, Eskisehir, Turkey

'Department of Emergency Medicine, Canakkale State Hospital, Canakkale, Turkey

${ }^{\mathrm{d} C}$ Corresponding Author: Ahmet Tugrul Zeytin, Yenidogan Mh. Sehit Serkan Ozaydin Sokak No:1 26060 Odunpazari, Eskisehir, Turkey.

Email: atzeytin@gmail.com
}

doi: http://dx.doi.org/10.14740/jmc2142w examination, the hip joint was at approximately $140^{\circ}$ flexion and rotation, and the knee joint was flexed. The patient was unable to move the hip joint. The patient did not have motor or sensory deficits and peripheral pulses were equally palpable in both lower limbs. Antero-posterior hip radiographs revealed inferior dislocation of the femoral head and the femur was flexed (Fig. 1). A closed reduction was performed after achieving sedation and analgesia with fentanyl and midazolam administration in the emergency room. The patient did not develop neurovascular deficit after the reduction. Control radiographs confirmed successful reduction of the femoral head (Fig. 2). The patient was moved to the Department of Orthopedics and Traumatology for follow-up.

\section{Discussion}

Inferior hip dislocation (luxatio erecta femoris) is an extremely rare clinical entity in adults. The incidence is higher in children due to the increased joint laxity and acetabulum containing a higher amount of unossified cartilage. In 1994, Beauchesne et al found a total of 24 case reports in the literature [3]. In the

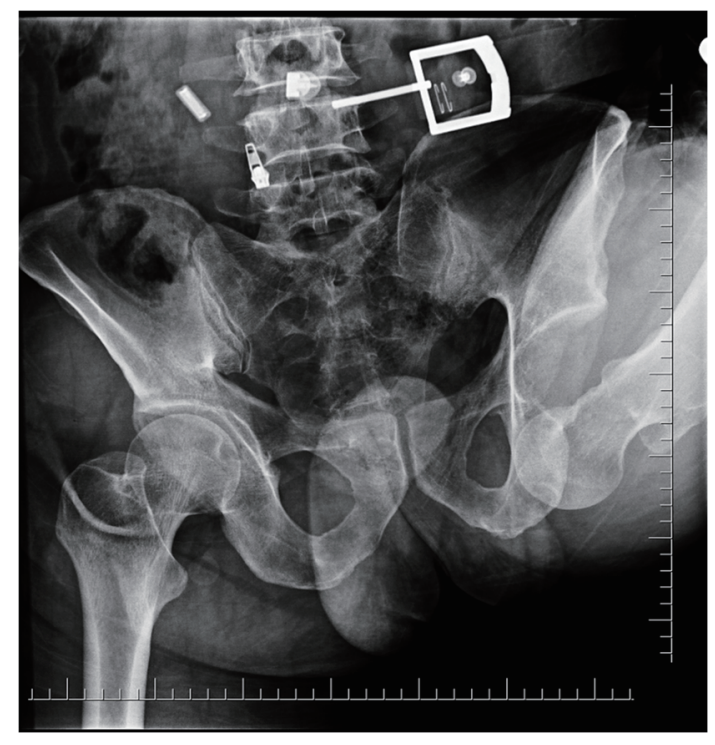

Figure 1. X-ray of the pelvis of the patient before reduction. 


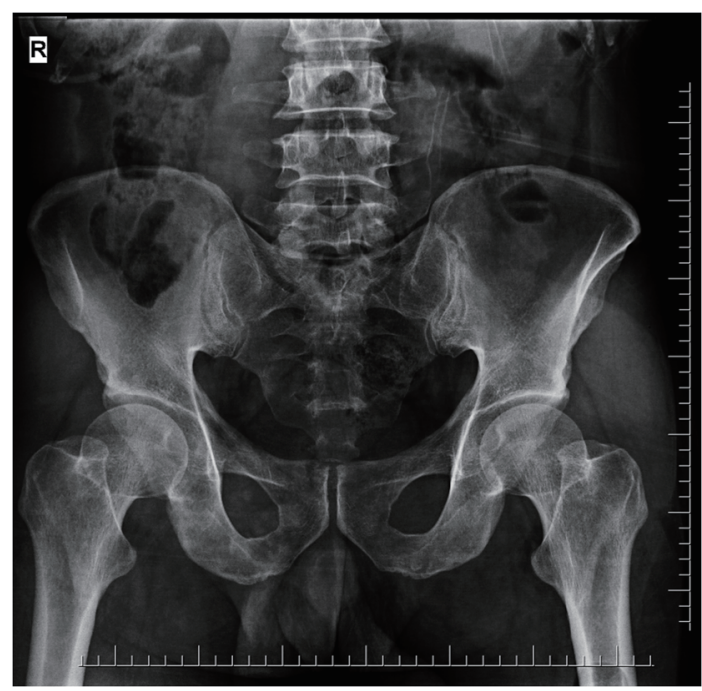

Figure 2. X-ray of the pelvis after reduction.

English literature, six cases were reported: five children and one adult. In the following period, the researchers of the current study found 10 reports of cases with inferior hip dislocation in the literature [2, 4-6].

Two injury mechanisms have been defined for the development of inferior hip dislocation. According to these mechanisms, inferior hip dislocation is divided into two sub-groups. In rare cases (inferior ischial dislocation and concurrent femoral inversion), the person falls on the flexed knee while the hip is in flexion. The weight of the body and the energy of the fall are transmitted to the femur. This energy is amplified by the flexion of the body. The femur head is dislocated from the inferior edge of the acetabulum. In more frequent cases (femoral inversion with concurrent anterior obturator dislocation), hip dislocation occurs with the femur abducted and externally rotated as a result of a fall or application of an external force to the femur. The femur head is dislocated inferior to the acetabulum as a result of strong abduction and external rotation and concurrent flexion toward the pelvis and the abdomen [7]. The mechanism of the frequent form explains the cause of injury in the present patient.

Inferior hip dislocation can be treated with closed reduction under sedation or general anesthesia. The reduction is performed with cephalad traction and extension and sometimes internal rotation. To date, fracture or neurovascular complication associated with the reduction has not been reported in the literature [6]. The current patient did not develop any complications. The complications reported in the literature mostly occurred in the late period, and there is one reported case of recurrent dislocation and another case developed avascular necrosis of femur head $[3,7]$.

\section{Conclusion}

It must be kept in mind that as a rare form of hip dislocation, inferior hip dislocation is not specific to the pediatric population and can also occur in adults. It is estimated that the incidence will be higher in the future due to the increasing incidence of high energy traffic accidents. The diagnosis is based on the typical extremity position and radiographs. A closed reduction can be performed with sedation and analgesia by applying gentle extension and internal rotation with concurrent cephalad traction.

\section{References}

1. Bartley RE, 3rd, Dimon JH, 3rd. Traumatic inferior hip dislocation in an adult (luxatio erecta). Orthopedics. 1995;18(12):1173-1174.

2. Singh R, Sharma SC, Goel T. Traumatic inferior hip dislocation in an adult with ipsilateral trochanteric fracture. J Orthop Trauma. 2006;20(3):220-222.

3. Beauchesne R, Kruse R, Stanton RP. Inferior dislocation (luxatio erecta) of the hip. Orthopedics. 1994;17(1):7274; discussion 74-75.

4. Walther MM, McCoin NS. Luxatio erecta of the hip. J Emerg Med. 2013;44(5):985-986.

5. Singh AP, Singh AP, Mittal V. Traumatic inferior hip dislocation with ipsilateral open subtrochanteric fracture: a rare case. Injury Extra. 2008;39:384-385.

6. Aggarwal S, Kumar V, Bhagwat KR, Shashikanth VS, Ravikumar HS. Inferior dislocation of the hip: a case series and literature review. Chin J Traumatol. 2012;15(5):317320 .

7. Brogdon BG, Woolridge DA. Luxatio erecta of the hip: a critical retrospective. Skeletal Radiol. 1997;26(9):548552. 